TAIWANESE JOURNAL OF MATHEMATICS

Vol. 13, No. 3, pp. 943-949, June 2009

This paper is available online at http://www.tjm.nsysu.edu.tw/

\title{
METRICAL TRANSITIVITY AND NONSEPARABLE EXTENSIONS OF INVARIANT MEASURES
}

\author{
A. B. Kharazishvili
}

\begin{abstract}
Under the Continuum Hypothesis, it is proved that any nonzero $\sigma$-finite metrically transitive invariant measure on a group of cardinality continuum admits a nonseparable invariant extension. An application of this result to the left Haar measure on a $\sigma$-compact locally compact topological group of the same cardinality is also presented.
\end{abstract}

Various left (right) invariant extensions of the left (right) Haar measure given on a Polish locally compact group are known in the literature. For instance, some nonseparable left invariant extensions of the left Haar measure on an infinite compact metrizable group is thoroughly considered in the monograph by Hewitt and Ross [1]. The construction of those extensions imitates the remarkable construction of Kakutani and Oxtoby [2] which was done in a particular case, for the Lebesgue measure on the one-dimensional unit torus. Clearly, the same construction works for the Lebesgue measure on the $n$-dimensional Euclidean space $\mathbf{R}^{n}$, where $n \geq 1$, and, more generally, for the left Haar measure on an uncountable locally compact Polish group.

It seems at first sight that specific topological properties of the Haar (respectively, Lebesgue) measure play an important role in the above-mentioned constructions. However, we will demonstrate in the sequel that the situation is absolutely different. The main goal of this note is to show that topological concepts are inessential for constructing nonseparable invariant extensions of an invariant measure given on a group of cardinality continuum, and the key role is played by the metrical transitivity of this measure (the precise definition of metrical transitivity is formulated below).

All measures considered in the present paper are assumed to be diffused (continuous), i.e. all of them vanish at singletons. This assumption is necessary for our further considerations (cf. Example 2 in the end of the paper).

Received January 12, 2007, accepted September 29, 2007.

Communicated by Yongsheng Han.

2000 Mathematics Subject Classification: 28A05, 28 D05.

Key words and phrases: Continuum Hypothesis, Invariant measure, Haar measure, Metrical transitivity, Nonseparable extension of measure, Ulam transfinite matrix. 
Let $E$ be a set, $G$ be a group of transformations of $E$ and let $\mu$ be a nonzero $\sigma$-finite $G$-invariant (more generally, $G$-quasiinvariant) measure on $E$.

Recall that $\mu$ is metrically transitive (or ergodic) if, for every $\mu$-measurable set $X \subset E$ with $\mu(X)>0$, there exists a countable family $\left\{g_{i}: i \in I\right\} \subset G$ such that

$$
\mu\left(E \backslash \cup\left\{g_{i}(X): i \in I\right\}\right)=0 .
$$

It is well known that the metrical transitivity (or ergodicity) of an invariant measure $\mu$ is closely connected with its uniqueness property. Indeed, if $\mu$ has the uniqueness property, then $\mu$ is necessarily metrically transitive. Conversely, if $G$ is uncountable and acts freely in $E$ and $\mu$ is complete, then the metrical transitivity of $\mu$ turns out to be equivalent to the uniqueness property (for more details, see [3]).

Example 1. The left Haar measure $\mu$ on a $\sigma$-compact locally compact group $G$ is metrically transitive. More precisely, let $H$ be any everywhere dense subgroup of $G$. Then the same $\mu$ considered as an invariant measure with respect to $H$ (which acts on $G$ from the left) is also metrically transitive (see, for instance, [3]).

A set $Y \subset E$ is called to be almost $G$-invariant with respect to $\mu$ if the equality $\mu(g(Y) \triangle Y)=0$ holds for all transformations $g \in G$. Clearly, $Y \subset E$ is almost $G$-invariant with respect to $\mu$ if and only if $E \backslash Y$ is almost $G$-invariant with respect to $\mu$. Moreover, the union of a countable family of almost $G$-invariant sets with respect to $\mu$ is almost $G$-invariant with respect to $\mu$. Therefore, the class of all almost $G$-invariant sets with respect to $\mu$ forms a $G$-invariant $\sigma$-algebra of subsets of $E$.

Below, the symbols $\mu_{*}$ and $\mu^{*}$ denote, respectively, the inner measure associated with $\mu$ and the outer measure associated with the same $\mu$.

A set $Z \subset E$ is called to be thick with respect to $\mu$ if $\mu_{*}(E \backslash Z)=0$.

For our further purposes, we need two auxiliary propositions.

Lemma 1. Let a measure $\mu$ be complete and metrically transitive (ergodic) and let a set $Y \subset E$ be almost $G$-invariant with respect to $\mu$. Then at least one of the following three relations is valid:

(1) $\mu(Y)=0$;

(2) $\mu(E \backslash Y)=0$;

(3) both sets $Y$ and $E \backslash Y$ are thick with respect to $\mu$ (consequently, both of them are nonmeasurable with respect to $\mu$ ).

This lemma is well known and its proof is omitted.

As usual, we denote by $\omega$ the first infinite cardinal, by $\omega_{1}$ the first uncountable cardinal and by $\mathbf{c}$ the cardinality of the continuum. Moreover, $\omega$ (respectively, $\omega_{1}$ 
and c) will be identified with the least ordinal number whose cardinality is equal to $\omega$ (respectively, $\omega_{1}$ and $\mathbf{c}$ ).

Let $U$ be a set of cardinality $\omega_{1}$. Recall that a double family $\left\{U_{n, \xi}: n<\omega, \xi<\right.$ $\left.\omega_{1}\right\}$ of subsets of $U$ is an Ulam matrix over $U$ if the following two conditions are satisfied:

(a) for any $n<\omega$, the family $\left\{U_{n, \xi}: \xi<\omega_{1}\right\}$ is disjoint;

(b) for any $\xi<\omega_{1}$, the set $U \backslash \cup\left\{U_{n, \xi}: n<\omega\right\}$ is at most countable.

The existence of Ulam matrices was first proved by Ulam in his classical work [6] (see also [5]). His result directly implies that $\omega_{1}$ is not a real-valued measurable cardinal. Some applications of Ulam matrices to the theory of invariant and quasiinvariant measures are presented in [3].

The next lemma is crucial for our further considerations.

Lemma 2. Let $\operatorname{card}(E)=\omega_{1}$ and let $\operatorname{card}(G) \leq \omega_{1}$. Suppose that $\mu$ is a nonzero $\sigma$-finite $G$-quasiinvariant metrically transitive measure on $E$. Then there exists a disjoint family $\left\{Y_{\xi}: \xi<\omega_{1}\right\}$ of subsets of $E$ such that:

(1) each set $Y_{\xi}\left(\xi<\omega_{1}\right)$ is thick with respect to $\mu$;

(2) for any $\Xi \subset \omega_{1}$, the set $\cup\left\{Y_{\xi}: \xi \in \Xi\right\}$ is almost $G$-invariant with respect to $\mu$.

Proof. We may assume, without loss of generality, that the given measure $\mu$ is complete. Consider the partition $\left\{O_{i}: i \in I\right\}$ of $E$ into $G$-orbits. Only two cases are possible.

1. All $G$-orbits are of $\mu$-measure zero. In this case, we necessarily have $\operatorname{card}(I)=$ $\omega_{1}$ (because our measure $\mu$ is nonzero). Let $\left\{I_{n, \xi}: n<\omega, \xi<\omega_{1}\right\}$ denote an Ulam matrix over $I$. We define

$$
Z_{n, \xi}=\cup\left\{O_{i}: i \in I_{n, \xi}\right\}\left(n<\omega, \xi<\omega_{1}\right) .
$$

Taking into account conditions (a) and (b), we easily get the following relations:

(c) for each $n<\omega$, the family $\left\{Z_{n, \xi}: \xi<\omega_{1}\right\}$ is disjoint;

(d) for each $\xi<\omega_{1}$, the set $E \backslash \cup\left\{Z_{n, \xi}: n<\omega\right\}$ is of $\mu$-measure zero.

It follows from (c) and (d) that there exist a natural number $n$ and an uncountable set $\Xi \subset \omega_{1}$ such that the inequality $\mu^{*}\left(Z_{n, \xi}\right)>0$ is satisfied for all $\xi \in \Xi$. Further, since our measure $\mu$ is metrically transitive and all sets $Z_{n, \xi}(\xi \in \Xi)$ are $G$-invariant, we can conclude (in view of Lemma 1) that 
all sets $Z_{n, \xi}(\xi \in \Xi)$ are also thick with respect to $\mu$. Let $\phi: \omega_{1} \rightarrow \Xi$ be a bijection. Putting

$$
\left\{Y_{\xi}: \xi<\omega_{1}\right\}=\left\{Z_{n, \phi(\xi)}: \xi<\omega_{1}\right\}
$$

we come to the required family of subsets of $E$.

2. There exists at least one index $i_{0} \in I$ for which we have $\mu^{*}\left(O_{i_{0}}\right)>0$. In this case, we obviously have

$$
\operatorname{card}\left(O_{i_{0}}\right)=\omega_{1}, \operatorname{card}(G)=\omega_{1} .
$$

Also, by Lemma 1 , the orbit $O_{i_{0}}$ is thick with respect to our measure $\mu$. Let us denote $V=O_{i_{0}}$ and let us fix a point $x \in V$. Clearly, the group $G$ acts transitively on $V$, i.e. $V=G(x)$. Let us represent $G$ in the form $G=\cup\left\{G_{\zeta}: \zeta<\omega_{1}\right\}$, where $\left\{G_{\zeta}: \zeta<\omega_{1}\right\}$ is an increasing (by inclusion) family of subgroups of $G$ satisfying the relations:

(e) $G_{\zeta} \neq \cup\left\{G_{\eta}: \eta<\zeta\right\}$ for all $\zeta<\omega_{1}$;

(f) $\operatorname{card}\left(G_{\zeta}\right) \leq \omega$ for all $\zeta<\omega_{1}$.

The existence of such a representation of $G$ is well known (see, for instance, [1], [2], [3] or [5]). This representation immediately yields us the relation

$$
V=G(x)=\cup\left\{G_{\zeta}(x): \zeta<\omega_{1}\right\} .
$$

Now, putting

$$
V_{\zeta}=G_{\zeta}(x) \backslash \cup\left\{G_{\eta}(x): \eta<\zeta\right\}
$$

for any $\zeta<\omega_{1}$, we see that $\left\{V_{\zeta}: \zeta<\omega_{1}\right\}$ is a disjoint covering of $V$ by countable sets. In addition, for every subset $\Theta$ of $\omega_{1}$, the set $\cup\left\{V_{\theta}: \theta \in \Theta\right\}$ is almost $G$-invariant with respect to the given measure $\mu$.

Consider an Ulam matrix $\left\{U_{n, \xi}: n<\omega, \xi<\omega_{1}\right\}$ over the set $U=\omega_{1}$ and define

$$
Z_{n, \xi}=\cup\left\{V_{\zeta}: \zeta \in U_{n, \xi}\right\} \quad\left(n<\omega, \xi<\omega_{1}\right) .
$$

Again, taking into account conditions (a) and (b), we infer that:

(g) for each $n<\omega$, the family of sets $\left\{Z_{n, \xi}: \xi<\omega_{1}\right\}$ is disjoint;

(h) for each $\xi<\omega_{1}$, the set $V \backslash \cup\left\{Z_{n, \xi}: n<\omega\right\}$ is at most countable and, consequently, the set $\cup\left\{Z_{n, \xi}: n<\omega\right\}$ is thick with respect to $\mu$.

It follows from $(\mathrm{g})$ and $(\mathrm{h})$ that there exist a natural number $n$ and an uncountable set $\Xi \subset \omega_{1}$ such that the inequality $\mu^{*}\left(Z_{n, \xi}\right)>0$ is valid for all ordinals $\xi \in \Xi$. Using the ergodicity of $\mu$, we deduce with the aid of Lemma 1 that all 
sets $Z_{n, \xi}(\xi \in \Xi)$ are thick with respect to $\mu$. Let $\phi: \omega_{1} \rightarrow \Xi$ be a bijection. Putting again

$$
\left\{Y_{\xi}: \xi<\omega_{1}\right\}=\left\{Z_{n, \phi(\xi)}: \xi<\omega_{1}\right\}
$$

we obtain the required family of subsets of $E$. Lemma 2 has thus been proved. The next auxiliary statement is a direct consequence of Lemma 2.

Lemma 3. Let $\operatorname{card}(E)=\mathbf{c}$ and let $\operatorname{card}(G) \leq \mathbf{c}$. Suppose that $\mu$ is a nonzero $\sigma$-finite $G$-invariant metrically transitive measure on $E$. Then, assuming the Continuum Hypothesis, there exists a disjoint family $\left\{Y_{\xi}: \xi<\mathbf{c}\right\}$ of subsets of E such that:

(1) each set $Y_{\xi}(\xi<\mathbf{c})$ is thick with respect to $\mu$;

(2) for any $\Xi \subset \mathbf{c}$, the set $\cup\left\{Y_{\xi}: \xi \in \Xi\right\}$ is almost $G$-invariant with respect to $\mu$.

Theorem 1. Under the assumptions of Lemma 3, there exists a nonseparable $G$-invariant extension $\mu^{\prime}$ of $\mu$. More precisely, the Hilbert dimension of the space $L_{2}\left(\mu^{\prime}\right)$ is equal to $2^{\mathbf{c}}$.

Proof. We preserve the notation of Lemma 3. Take a family $\left\{Y_{\xi}: \xi<\mathbf{c}\right\}$ as in that lemma. As known (see, e.g., [1] or [2]), there exists a family $\left\{\Xi_{j}: j \in J\right\}$ of subsets of $\mathbf{c}$ such that:

(1) $\operatorname{card}(J)=2^{\mathbf{c}}$;

(2) for any injective sequence $\left\{j_{k}: k<\omega\right\} \subset J$, we have

$$
\operatorname{card}\left(\cap\left\{\Xi_{j_{k}}^{\prime}: k<\omega\right\}\right)=\mathbf{c},
$$

where $\Xi_{j_{k}}^{\prime}=\Xi_{j_{k}}$ or $\Xi_{j_{k}}^{\prime}=\mathbf{c} \backslash \Xi_{j_{k}}$.

Let us define

$$
A_{j}=\cup\left\{Y_{\xi}: \xi \in \Xi_{j}\right\}(j \in J) .
$$

In this way we come to the family $\left\{A_{j}: j \in J\right\}$ of almost $G$-invariant subsets of $E$ which are $\sigma$-independent (in the generalized sense) with respect to $\mu$. The last phrase means that, for any injective sequence $\left\{j_{k}: k<\omega\right\} \subset J$, we have

$$
\mu_{*}\left(E \backslash \cap\left\{A_{j_{k}}^{\prime}: k<\omega\right\}\right)=0,
$$

where $A_{j_{k}}^{\prime}=A_{j_{k}}$ or $A_{j_{k}}^{\prime}=E \backslash A_{j_{k}}$.

Now, applying the standard method of extending measures (see [1], [2]), we can extend our $\mu$ to a $G$-invariant measure $\mu^{\prime}$ so that all sets from the family $\left\{A_{j}: j \in J\right\}$ become $\mu^{\prime}$-measurable. Moreover, the above-mentioned method guarantees that the Hilbert dimension of the space $L_{2}\left(\mu^{\prime}\right)$ is equal to $2^{\mathbf{c}}$ (cf. [1], [2]). This completes the proof of Theorem 1. 
Remark 1. Unfortunately, the obtained extension $\mu^{\prime}$ is not metrically transitive, because all sets $A_{j}(j \in J)$ are almost $G$-invariant with respect to $\mu^{\prime}$. It would be interesting to find a general method of extending measures by means of which a given metrically transitive invariant (respectively, quasiinvariant) measure can be extended to a nonseparable invariant (respectively, quasiinvariant) metrically transitive measure.

In this context, it must be noticed that the method described in [4] by Kodaira and Kakutani also leads to a nonseparable translation-invariant extension of the Lebesgue measure and this extension possesses the property of metrical transitivity. But the construction given in [4] is very special and we do not know any purely measure-theoretical version of the method suggested by Kodaira and Kakutani.

Theorem 1 directly implies the following statement.

Theorem 2. Assume the Continuum Hypothesis. Let $G$ be a $\sigma$-compact locally compact group of cardinality $\mathbf{c}$ and let $\mu$ be a left invariant metrically transitive extension of the left Haar measure on $G$. Then there exists a left invariant extension $\mu^{\prime}$ of $\mu$ such that the Hilbert dimension of the space $L_{2}\left(\mu^{\prime}\right)$ is equal to $2^{\mathbf{c}}$.

Remark 2. It should be mentioned that a measure $\mu$ in Theorem 2 can be nonseparable itself (in this connection, see, e.g., [3]). This circumstance also indicates that Theorem 2 cannot be established by using the methods developed in [1] and [2].

Remark 3. As pointed out in the beginning of the paper, the construction of Kakutani and Oxtoby [2] (as well as its generalization presented in the monograph [1]) is based on special topological properties of the Lebesgue (respectively, Haar) measure. On the other hand, Theorem 1 shows that, replacing those properties by the metrical transitivity (ergodicity), we can obtain a much stronger assertion. However, the Continuum Hypothesis was essentially used in our argument and, at this moment, we do not know whether additional set-theoretical assumptions are necessary for obtaining the above-mentioned stronger result. We even do not know whether Theorem 1 remains true under Martin's Axiom instead of the Continuum Hypothesis. Recall that Martin's Axiom is much weaker than the Continuum Hypothesis and does not restrict (from above) the cardinality of the continuum.

Example 2. Let $E$ be a set of cardinality $\mathbf{c}$ and let $E^{\prime}$ be an infinite countable subset of $E$. Define $G$ as the group of all those transformations of $E$ whose restrictions to the set $E \backslash E^{\prime}$ coincide with the identical transformation of $E \backslash E^{\prime}$. Obviously, $\operatorname{card}(G)=$ c. Further, define a measure $\nu$ on the power set of $E$ by putting $\nu(Z)=\operatorname{card}\left(Z \cap E^{\prime}\right)$ if $\operatorname{card}\left(Z \cap E^{\prime}\right)<\omega$ and $\nu(Z)=+\infty$ if 
$\operatorname{card}\left(Z \cap E^{\prime}\right) \geq \omega$. It can easily be verified that $\nu$ is $\sigma$-finite, $G$-invariant, metrically transitive and nonseparable. If $\nu_{1}$ denotes a probability measure equivalent to $\nu$, then $\nu_{1}$ is separable and $G$-quasiinvariant. At the same time, $\nu_{1}$ has no proper extension because the domain of $\nu_{1}$ is the whole power set of $E$. In particular, $\nu_{1}$ does not admit a nonseparable $G$-quasiinvariant extension.

This simple example shows that the assumption of the diffusedness of a measure $\mu$ in the formulation of Theorem 1 is rather essential for the validity of this theorem.

In a similar way, we can define continuumly many probability measures on the powerset of $E$, which are $G$-quasiinvariant, metrically transitive and separable. Similarly to $\nu_{1}$, those measures do not admit nonseparable $G$-quasiinvariant extensions.

\section{REFERENCES}

1. E. Hewitt and K. A. Ross, Abstract Harmonic Analysis, Vol. 1, Springer-Verlag, Berlin-New York, 1963.

2. S. Kakutani and J. C. Oxtoby, Construction of a nonseparable invariant extension of the Lebesgue measure space, Ann. Math., 52 (1950), 580-590.

3. A. B. Kharazishvili, Transformation groups and invariant measures, World Scientific Publ. Co., London-Singapore, 1998.

4. S. Kodaira, S. Kakutani, A nonseparable translation-invariant extension of the Lebesgue measure space, Ann. Math., 52 (1950), 574-579.

5. J. C. Oxtoby, Measure and category, Springer-Verlag, Berlin-New York, 1971.

6. S. Ulam, Zur Masstheorie in der allgemeinen Mengenlehre, Fund. Math., 16 (1930), 140-150.

A. B. Kharazishvili

A. Razmadze Mathematical Institute,

1, M. Alexidze Street,

Tbilisi 0193,

Georgia

I. Chavchavadze State University,

32, I. Chavchavadze Street,

Tbilisi 0128,

Georgia

E-mail:kharaz2@yahoo.com 